\title{
Multisensor Optical-Electronic Device for Calculation of Surface Parameters of the 3D Curvelinear Object
}

\author{
Konstantin KARAVAEV, Maxim I. TRUPHANOV \\ Silver Research Laboratory, Los Angeles (CA), USA \\ DOI: $10.15221 / 16.279 \quad$ http://dx.doi.org/10.15221/16.279
}

\begin{abstract}
The research covers the operation principles and structure functional organization of specialized optical-electronic device for calculation of a volumetric object.

The purpose of the device is to build the 3D plane, which with the given degree of precision approximates the surface of the top and nape sections of a human head intended for implication in the production of customized headwear and elements of protectiveclothing or for other similar tasks related to the analysis of the 3D objectshape.

The proposed device provides the ability to build the 3D model of an object under study by acquiring and analyzing the series of images of its surface continuously acquired from three or four optical-electronic sensors throughout the measurement process. The number of the optical-electronic sensors depends on the configuration of the optical-electronic device. Optical-electronic sensors organized in a given order relative to each other constitute a unified system, which in its turn is to be placed in the prescribed range of positions in relation to the head.

In order to simplify the procedure and enhance the accuracy of calculation of special coordinates for a set of points of the object surface and reduce the computational complexity of the developed algorithms we use the tight-fitting contact hat consisting of a set of special contrast markers., which is to be put on the head.

The following beneficial features of the proposed method and the optical-electronic device have the following beneficial features: (i) no strict requirements to interrelated positioning of the analyzed object and the system of the optical-electronic sensors; (ii) possibility to use commercially available web cameras as optical-electronic sensors; and (iii) no structurally complex and moving mechanical elements, which allow its mass use with no reduction in the accuracy of calculated point coordinates on the surface of an object required for practical tasks.

The experiments we conducted showed that depending on the interrelated position of the device and the analyzed object our optical-electronic device gives the calculation error of the 3D coordinates of about \pm 2 to $\pm 7 \mathrm{~mm}$, which is sufficient for practical tasks.
\end{abstract}

Keywords: 3d body scanning, binocular vision, machine vision, distortion.

\section{Introduction}

One of the key tasks in the manufacturing various customized ware (clothes, footwear, protective outerwear) for personal use is to calculate in a short time the parameters of the 3D shape of body parts with precision sufficient for practical implementation. In our case the required accuracy is determined by a specific task and ranges, generally, from $3 \mathrm{~mm}$ to $7 \mathrm{~mm}$. In turn, the task time,depends on the readiness of a testee to participate in the measurements, generally, it should be about $30-40 \mathrm{~s}$ at most. Evidently, the time it takes to measure the form of the surfaces is foremost determined by the measuring method.

The proposed optical-electronic device implements the contactless way of measuring the parameters of a human head, and it is intended, primarily, for calculation of the parameters of the head.

Further, we provide a description of the mathematical framework for the operation of our device and its structure-functional analysis.

\section{Mathematical framework for operation of proposed optical-electronic device}

The operation principle of our device and calculation of parameters of the 3D object shape in a three-dimensional space consist of the following steps: 
(1) placing of the system of four (three) video cameras around the object in a prescribed order. (The analysis of the head is performed with four cameras, the analysis of a leg or a part of body is performed with three cameras. Further in the paper we will consider four cameras since our device is intended for calculation of the parameters of the head);

(2) placing of the contact hat on the head;

(3) continuous acquisition of images from the cameras slightly turning and moving the head;

(4) iterative image processing including calculation of the 3D coordinates of point sets from data from each pair of images (stereopair) and specification of point coordinates observed simultaneously in several pairs of stereo images;

(5) tracking each point with every turn of the head and complexation of acquired data on 3D positions of the points to enhance the accuracy of calculation of their 3D positions;

(6) yielding the parameters of the surface of the head as a cloud of the 3D point coordinates on the object surface with separation of about $5 \mathrm{~mm}$ and accuracy $\pm 2 \mathrm{~mm}$ or as a set of elementary interconnected polygons that approximate the surface of the head.

In order to minimize the effect of exterior lights, we use stationary IR lighting, which provides all-round illumination of the object.

\subsection{Composition and structure of proposed optical-electronic device}

The proposed optical-electronic device is composed of:

- three (four) portable video cameras oriented in a prescribed position relative to each other (three cameras are used for extended objects, such as ankles or body parts; four cameras are used for objects with a near half-sphere shape, in our case, the head);

- system of seven flat small-sized mirrors which provide acquisition of additional images from each camera;

- computing aids for image processing based on PC or a computing block based on special image processing module.

Figure 1 illustrates the arrangement of video cameras, mirrorsand the analyzed object (the head). Figures 2(a) and 2(b) illustrate top view and the side view of the implemented system, correspondingly.

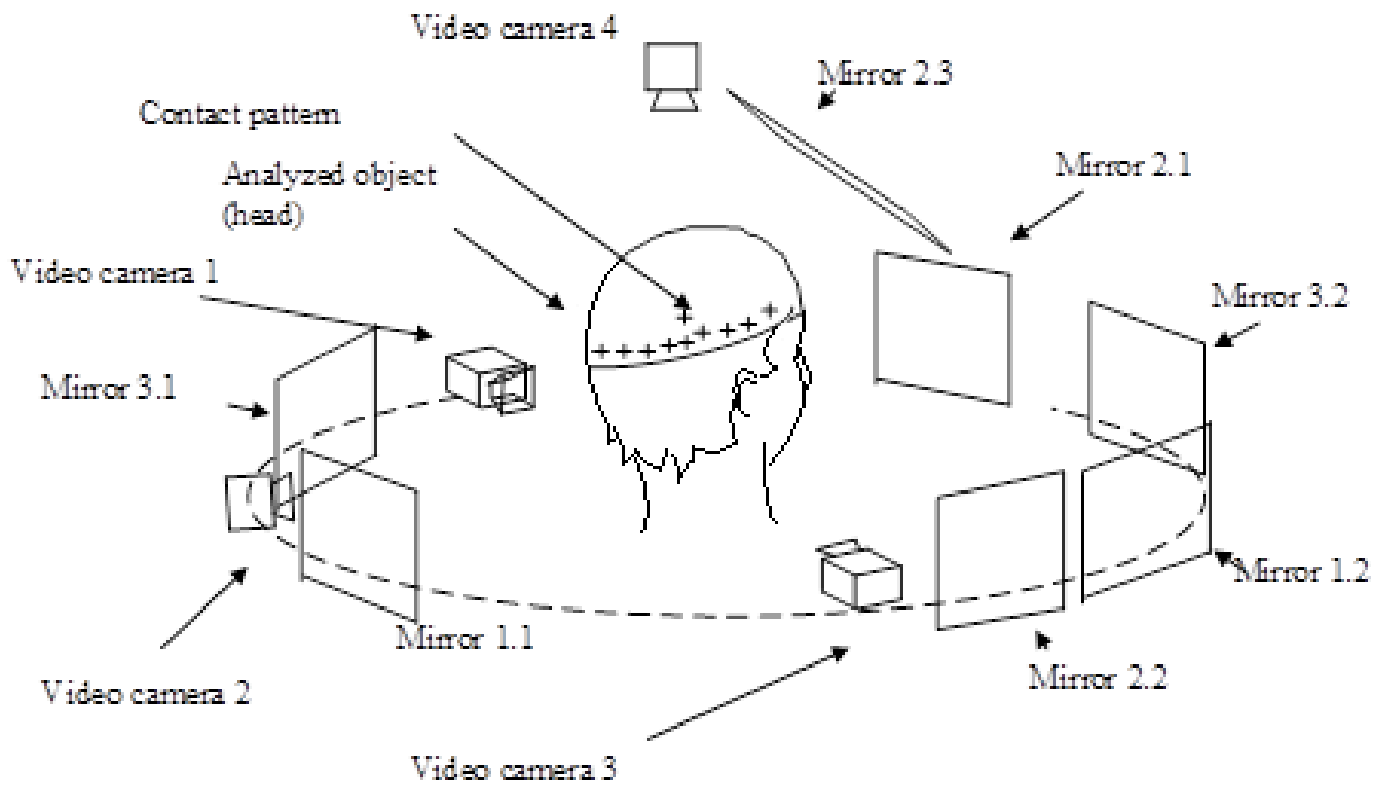

Fig. 1.Arrangement of elements of the optical-electronic device in relation to the analyzed object. 


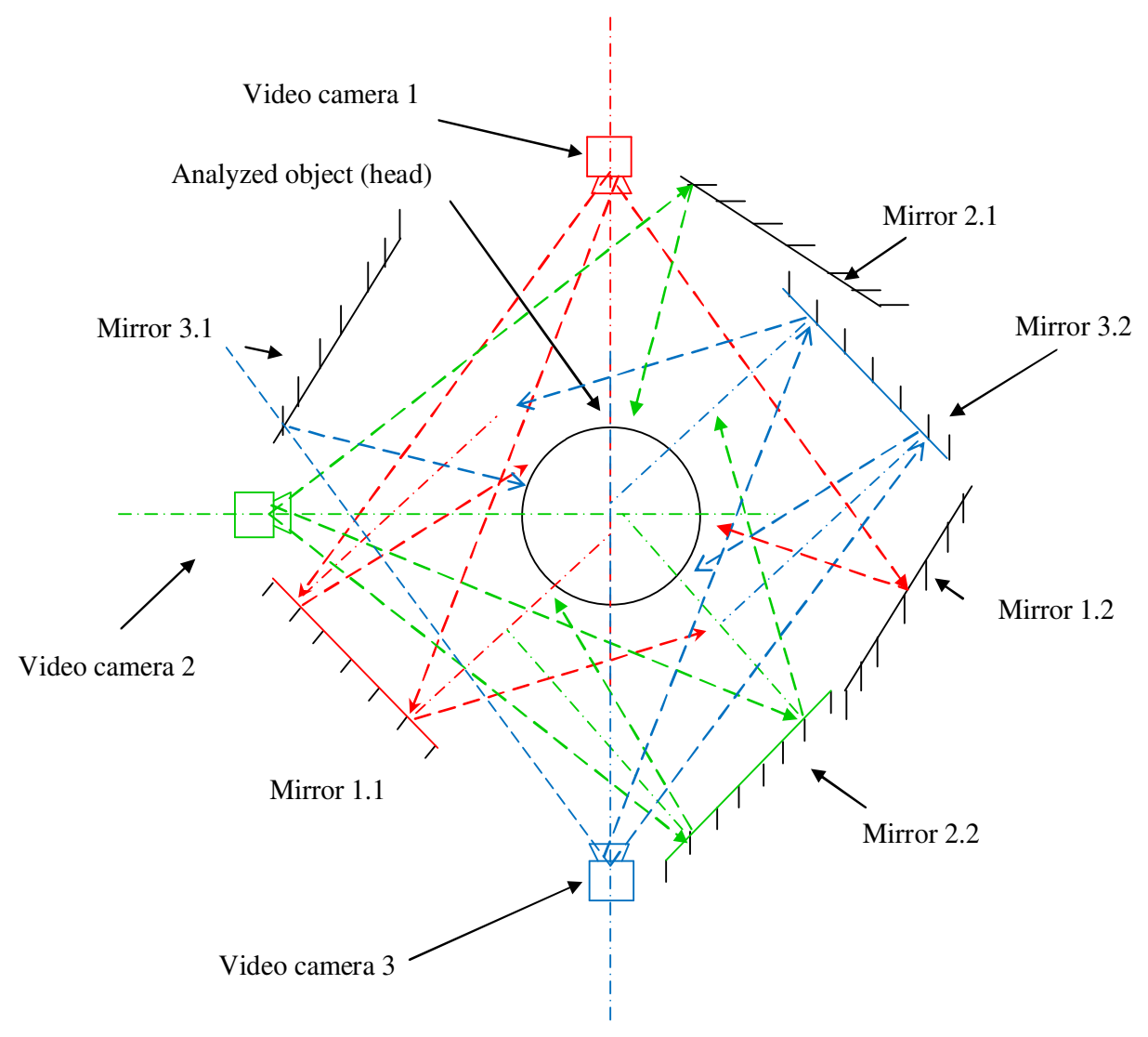

Fig. 2a.Arrangement of elements of the optical-electronic device in relation to the analyzed object (top view).

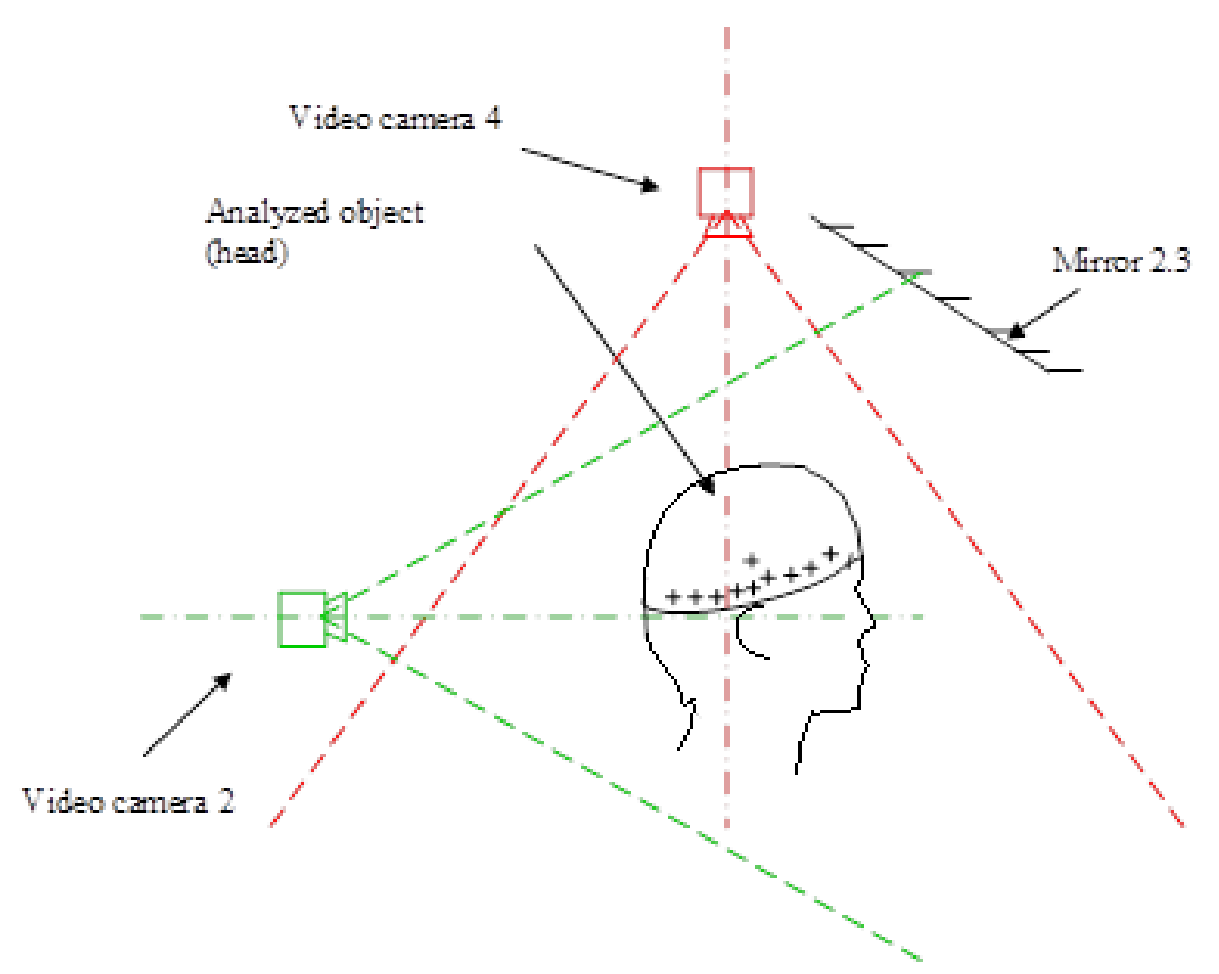

Fig. 2b.Arrangement of elements of the optical-electronic device in relation to the analyzed object (side view). 
Constructively, all optical-electric sensors and mirrors are fixed rigidly in relation to each other on predetermined positions which provide the ability to observe the same points on the object surface from several sides at the same time. This ability allows us to choose the stereo pair of images which will yield maximum accuracy in calculation of the $3 \mathrm{D}$ point coordinates.

Before operating the device, it must be calibrated according to the proposed procedures based on the method [3].

We should note, that the analyzed object must not be immobilized or rigidly fixed, it may obtain random position approximately in the center of the system illustrated in Figure 1. Also during image taking the object can move or turn slightly.

The computation module of the device used the developed algorithms to carry out the calculation of the 3D coordinates of individual fragments of the surface of the analyzed object and synthesize a united model of the object irrespective of the movement trajectory of the mobile platform.

\subsection{Mathematic model for calculation of object parameters in 3D space}

The mathematic model of the proposed optical-electronic device operation describes the operations for processing of input images and generation of the 3D surface of the analyzed object.

The mathematic model contains the following submodels:

- image preprocessing including the minimization of random noise and systematic distortions in the optical system;

- detection and registration of characteristic points on the object surface in different frames of the images from a stereo pair;

- tracking the characteristic points on the surface of the head when it is moving or turning;

- calculation of the 3D point coordinates on the surface of the analyzed object;

- generation of the 3D continuous plane as the set of simple triangles which vertices are the detected characteristic points.

\section{Submodel of image preprocessing}

In order toeliminate random noise from the acquired images we used the adaptive filtering with filtration coefficients which vary depending on the structure of the processed image [1]:

$$
I\left(n_{1}, n_{2}\right)=\sum_{k 1, \pi z=D} h\left(n_{1}, n_{2} ; k_{1}, k_{21}\right) g\left(n_{1}+k_{2} ; n_{2}+k_{2}\right)
$$

where $n_{1}, k_{1} \in\left[0, N_{1}\right], n_{2}, k_{2} \in\left[0, N_{2}\right], \quad$ are the samples of the field under observation; $\mathrm{H}$ is the normalizing filter coefficient, which ensures the unbiasedness of average brightness of the processed image compared to the original image; $D$ is the 2D discrete observation field of $N_{1} \times N_{2}$ dimension; $h\left(n_{1}\right.$, $n_{2}$ ) is the pulse characteristic, which determines the filtration parameters; $g\left(n_{1}, n_{2}\right)$ is the current local area of the image under processing.

Filter coefficients $h\left(n_{1}, n_{2} ; k_{1}, k_{2}\right)$ depend on the image brightness values in the sliding window $D$.

Next, we describe the correction of the most significant systematic distortion of the optical system, i.e. radial distortion. The model of radial distortion is written as the infinite series [2]:

$$
\begin{aligned}
& x_{u}=x_{d}\left(1+k_{1} r_{d}^{2}+k_{2} r_{d}^{4}+\ldots\right), \\
& y_{u}=y_{d}\left(1+k_{1} r_{d}^{2}+k_{2} r_{d}^{4}+\ldots\right) .
\end{aligned}
$$

In our research with use the novel approach tothe determination of distortion parameters without implication of special calibration object, introduced in[3]. According to this approach, the coefficients of radial and tangential distortion are determined during the straight-line movement of the calibrated optical-electronic sensor in relation to [3].The undistorted point coordinates are derived with the formula:

$$
\begin{aligned}
& x_{u}=x_{d}\left(1+k_{1} r_{d}^{2}\right), \\
& y_{u}=y_{d}\left(1+k_{1} r_{d}^{2}\right),
\end{aligned}
$$

where $r_{d}=\sqrt{x_{d}^{2}+y_{d}^{2}}$ is the distance from the center of the frame to the currently analyzed point in the image. 
The combined error of radial and tangential components of distortion are determined as:

$$
\begin{aligned}
& \delta_{x}=k_{1} x\left(x^{2}+y^{2}\right)+p_{1}\left(3 x^{2}+y^{2}\right)+2 p_{2} x y, \\
& \delta_{y}=k_{1} y\left(x^{2}+y^{2}\right)+p_{2}\left(3 x^{2}+y^{2}\right)+2 p_{1} x y,
\end{aligned}
$$

where $k_{1} x\left(x^{2}+y^{2}\right)$ and $k_{1} y\left(x^{2}+y^{2}\right)$ are the values of previously determined radial distortion; $p_{1}\left(3 x^{2}+y^{2}\right)+2 p_{2} x y$ and $p_{2}\left(3 x^{2}+y^{2}\right)+2 p_{1} x y$ are the values of tangential distortion; $\mathrm{p} 1, \mathrm{p} 2$ are the tangential distortion coefficients.

The distortion correction in the acquired images consists of moving all points in the image to their true positions, i.e. the positions which they would have occupied if there were no effect of radial distortion and acquisition of corrected images which are submitted for further processing.

\section{Submodel of identification of characteristic image points}

The basis of the model of identification of characteristic points is the implication of the approximated Hessian matrix determinant for extraction of special points. The values of the determinant are stored on special maps called the "response maps". The detectors based on the Hessian matrix determinant are known to provide best results in special points search tasks.

Given $\mathrm{X}(\mathrm{x}, \mathrm{y})$ is the point in the image $\mathrm{I}$, the Hessian matrix for this point is:

$$
H(x, \sigma)=\left[\begin{array}{ll}
L_{x x}(x, \sigma) & L_{x y}(x, \sigma) \\
L_{x y}(x, \sigma) & L_{x y}(x, \sigma)
\end{array}\right]
$$

where $L_{x x}(x, \sigma)$ is the Gaussian wrapping of the second order derivative of the image $I$ in the point $X$, analogously $L_{x y}(x, \sigma)$ and $L_{y y}(x, \sigma)$.

Implementation of the Gaussian function for scale-space image analysis is optimal [4].

In order to track the identified characteristic points during movements and turns of the head, we estimate the Euclidean distances between those special points in the feature space, which values are present in the descriptors of special points with consideration of the known direction of movement of a part of the analyzed surface. Then, depending on the value of the selected threshold, the decision of the similarity of the points is made. This requires calculation of the Euclidean distance $v$ between corresponding objects in the feature space as:

$$
r_{E}\left(o_{i}, o_{j}\right)=\sqrt{\sum_{n=0}^{m-1}\left(o_{m}-o_{m n}\right)^{2}}
$$

where $\vec{a}_{1}^{*}=\left(a_{i 0}, \ldots, a_{i 0 m-1}\right), \vec{o}_{3}=\left(a_{i 0}, \ldots, o_{j 0 m-1}\right)$ are the corresponding vectors which describe the features, $m$ is the dimensionality of the feature vector.

If the distance value satisfies the following expression, the objects are considered "similar":

$$
\mathrm{r}_{\mathrm{E}}\left(O_{i}, O_{j}\right)<\mathrm{p}_{\mathrm{e}} \text {, }
$$

where $p_{e}$ is the threshold determined experimentally.

\section{Submodel of calculation of the 3D characteristic points coordinates}

Here we introduce the arrangement model for one of the pairs of optical-electronic sensors, and the geometric model of building the projection of point $\mathrm{N}$ of the tested scene on the planes $P_{11}, P_{12}$ in the images from optical-electric sensors (Fig. 3). It should be noted that though the proposed device includes three optical-electronic sensors, here, to generate the 3D representation, we use two pairs of optical-electronic sensors which take images directly or by means of mirrors.

In order to realize the binocular vision, which provides the dimensional representation of the object placed in the work scene in sight of the optical-electronic device, and calculate the 3D coordinates on the object surface, we arrange the optical-electronic sensors with prior distance $L$ and shifted at the given angle in relation to each other. 


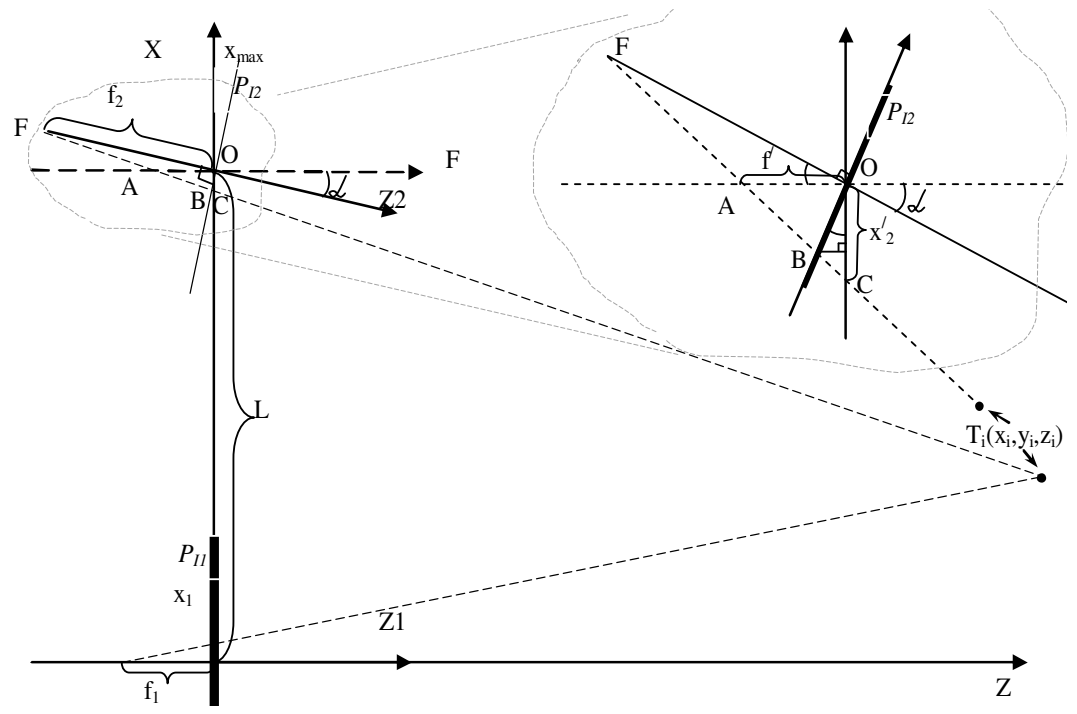

Fig. 3.Geometric model for calculating the $3 D$ coordinates of the point $T_{i}$ of the object in the work scene.

Figure 3 illustrates the geometric arrangement model of planes in the images from the optical-electronic sensors with random mutual orientation in the horizontal plane.

Model $\mathrm{M}_{\mathrm{C} 3 \mathrm{D}}$ for calculating the $3 \mathrm{D}$ coordinates $\left(\mathrm{Z}_{\mathrm{Fr}}, \mathrm{x}_{\mathrm{Fr}}, \mathrm{y}_{\mathrm{Fr}}\right)$ of the characteristic point $\mathrm{Fr}_{\mathrm{j}}$, determined by the method described in the previous paragraph, is

$$
<Z_{F r j} X_{F r j}, y_{F r j}>=M_{C 3 D}\left(x_{1 F r j}, x_{2 F r j}, y_{1 F r j}, y_{2 F r j}, f_{1}, f_{2}, x_{\max 1 p}, x_{\max 2 p}, y_{\max 1 p}, y_{\max 2 p}, L_{v s 1}, L_{v s 2}, L, \alpha\right),
$$

wherex $x_{1 F r}, X_{2 F r j}$ are the abscisses, and $y_{1 F r j}, y_{2 F r j}$ are the ordinates of the point $F r_{j}$ in the image from first and second optical-electronic devices,

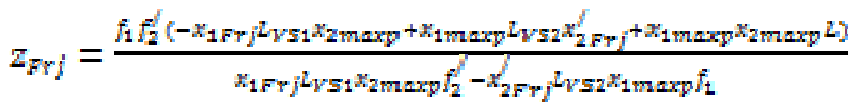

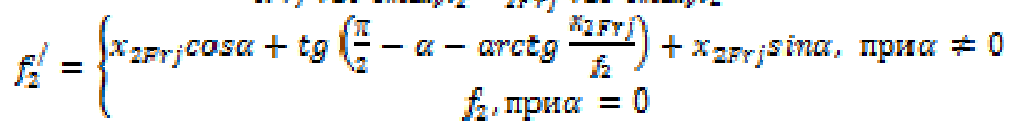

$$
\begin{aligned}
& x_{2 F Y j}^{l}=\left\{\begin{array}{c}
x_{2 F r j} \cos \alpha+\frac{x_{2 F r j} \sin \alpha}{t_{g}\left(\frac{\pi}{2}-\alpha-a r c t g\right.} \frac{\left.x_{7 F r}\right)}{f_{2}}, \pi p n \alpha \neq 0 \\
x_{2 F r j}, \pi p n \alpha=0
\end{array},\right. \\
& x_{F V j}=x_{1 F V} \frac{\left(\hat{A}+z_{F r j}\right)}{f}, \\
& y_{F r j}=y_{1 F j} \frac{\left(\hat{A}+z_{F r j}\right.}{f_{1}} \text {. }
\end{aligned}
$$

Expressions are used to calculate the 3D coordinates of identified characteristic points on the surface of the analyzed object.

Submodel MS for tracking the objects in the work scene is:

$$
<Q o b j>j \mid j=1 . . Q t=M S(\text { Qtprev, Fr, Fr_tprev), }
$$

where Qtprev denotes previously detected and tracked objects in the work scene from the previous frame, Fr are the parameters of registered characteristic points for current frames, Fr_tprev are the parameters of registered characteristic points from the previous frame.

Tracking the points during movements of the movable platform is performed in two stages. In the first stage, we calculate the coordinates of points with the sequences of frames from each optical-electronic sensor and store them into a special array. In the second stage, if the characteristic points have similar changes in their 3D coordinates, we associate the points from the new frame with the points from the previous frame; this completes tracking in this area. If changes in the point coordinates are not similar, and the histogram of the offset values distribution has more than one pronounced maximum, we make an assumption that registration of the point is unreliable and thus requires additional refinement followed by adjustment of coordinates of given area on the object surface by submitting the parameters to the submodel for object detection and repeating the calculation for object detection only in the local area, where the object was in the previous frame. 
In this section, we explained main mathematic models which describe the process of the proposed device operation.

\section{Functional structure of proposed optical-electronic device}

Figure 3 illustrates the functional structure of the proposed optical-electronic device for estimation of the 3D parameters of the analyzed object surface.

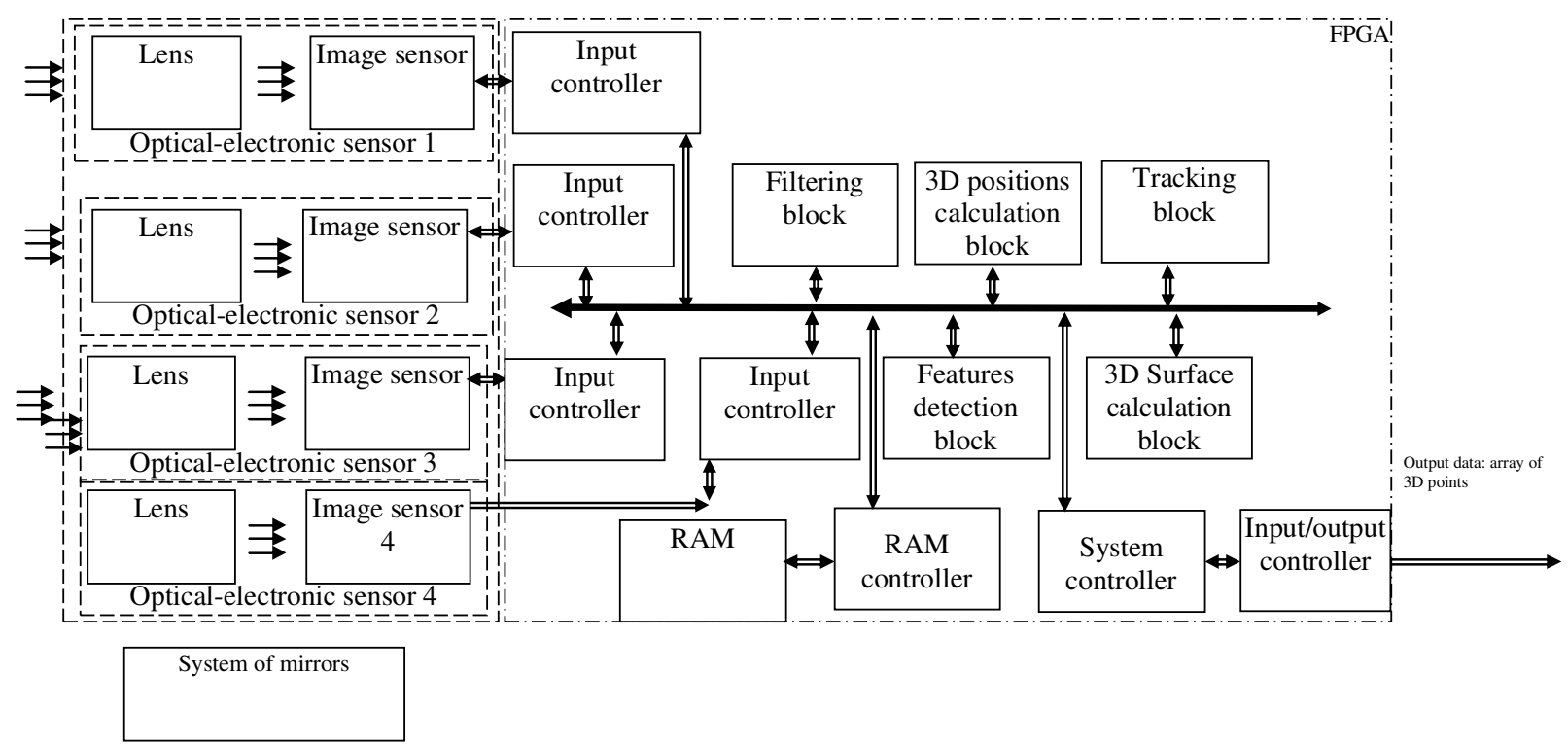

Fig. 3.Functional structure of the proposed optical-electronic device.

The proposed device consists of the following modules for image acquisition and processing: three optical-electronic sensors and FPGA module based on the programmable logic Xilinx Spartan 3, which realizes main stages of image processing according to the mathematic model presented in section 2 .

\section{Experimental results}

The results of the experiment we conducted with the model of the proposed optical electronic device proved the sufficiency and applicability of the proposed theoretical statements.

It was determined that during the analysis of the surface of the human head the error is about $\pm 4.48 \mathrm{~mm}$ with average of the $2.1 \mathrm{~mm}$. Indicated errors are sufficient for the stated practical tasks, i.e. design of individually customized headwear and individual protective clothing.

\section{References}

[1] A. Reza. Adaptive Noise Filtering of Image Sequences in Real Time Alim. Reza U.S. Coast Guard Academy Department of Engineering, Electrical Engineering Section 15 MoheganAve., NewLondon, CT 06320 U.S.A. Issue 4, Volume 12, April 2013, pp. 189-201.

[2] Tsai, R.Y. A versatile camera calibration technique for highaccuracy 3D machine vision metrology using off-the-shelf TV cameras and lenses. / Tsai, R.Y. // IEEE J Robotics Autom 3(4) - P. 323-344.

[3] V. Titov. The calibration method for stereoscopic vision system [text] / V. Titov, S. Degtiarev, M. Truphanov // Machine graphics and vision. Poland, Vol. 17, No. 4, 2008. - pp. $373-387 .$.

[4] Viksten, F. Point-of-Interest Detection for Range Data [Text] / Viksten, F. Nordberg, K. Kalms, M. // Pattern Recognition, 2008. ICPR 2008. 19th International Conference on Publication Date: 8-11 Dec. 2008, P.1-4 